\title{
Gemeinsam gegen rechts
}

\section{Die Freie Wohlfahrtspflege gegen pädagogische und soziale Bestrebungen rechtsextremer Organisationen}

\author{
Thomas Niermann und Mahmut Kural
}

Thomas Niermann ist beim

Gesamtverband des Paritätischen

Wohlfahrtsverbandes Abteilungsleiter

Soziale Arbeit und Internationale

Kooperation. Er ist langjähriges Mitglied im Beirat der Blätter der Wohlfahrtspflege.

E-Mail alsoz@dpwv.de

Mabmut Kural ist Referent für

Grundlagen der Jugendhilfe beim

Bundesverband des Deutschen Roten

Kreuzes und Vorsitzender der

Arbeitsgruppe »Rechtsextremismus « der Bundesarbeitsgemeinschaft der Freien

Wohlfahrtspflege.

E-Mail kuralm@drk.de
Rechtsextreme Organisationen interessieren sich zunehmend für die Übernahme von sozialen Beratungseinrichtungen und für die Mitgliedschaft in einem Spitzenverband der Freien Wohlfahrtspflege. Das vermeitlich neue Image des "sozialen Kümmeres" dient jedoch den alten Zwecken des Rechtsextremismus: einem übersteigerten Nationalismus mit Fremdenfeindlichkeit und Antisemitismus, einem autoritär-konservativen, hierarchisch geprägten Familien- und Gesellschaftsbild und der Ablehnung der Demokratie.

Der Rechtsextremismus stellt eine konstant hohe Gefahr für die Demokratie in Deutschland dar. Jenseits der gestiegenen und öffentlich wahrgenommenen Gewaltbereitschaft der rechtsextremen Szene, versuchen die Nationaldemokratische Partei Deutschlands (NPD) und ihre Vorfeldorganisationen sich zunehmend als sozial engagierte Alternative darzustellen. Ihre Funktionäre und Anhänger treten dabei betont gemäßigt und bürgernah auf. Anders sieht die Wirklichkeit aus: Jahr für Jahr werden über eintausend rechtsextremistisch motivierte Gewalttaten gezählt. Der Verfassungsschutz geht davon aus, dass von rund 31.000 Rechtsextremisten etwa 10.000 gewaltbereit sind.

Aber »Rechtsextreme sehen nicht immer so aus, wie man sie sich vorstellt. Sie sind auch nicht so dumpf, wie man sie gern hätte «, schrieb das Magazin »Campus « der Wochenzeitung »Die Zeit« im Frühjahr 2008. Rechtsextreme sitzen in drei Landtagen und zahlreichen Kommunalparlamenten. Sie nutzen Formen zivilen Engagements zur Verbreitung ihrer antidemokratischen Ideologie. Sie engagieren sich in Bürgerinitiativen ebenso wie in der Elternarbeit von Kindertageseinrichtungen oder Schulen. Rechtsextreme Organisationen interessieren sich für die Übernahme von Beratungseinrichtungen und für die Mitgliedschaft in einem
Spitzenverband der Freien Wohlfahrtspflege.

Seit dem Wechsel an der Parteispitze im Jahre 1996 versucht die NPD in der Öffentlichkeit weniger mit revisionistischen und neonazistischen Verlautbarungen aufzufallen, sondern sich zunehmend zu sozialen Themen zu positionieren. Die Partei arbeitet seither nach einer aggressiven Drei-Säulen-Strategie: »Kampf um die Straße, Kampf um die Parlamente, Kampf um die Köpfe«, die sie inzwischen zu einem Vier-Säulen-Konzept erweitert hat. Dieser mehr strategischen denn ideologischen Umorientierung Mitte der 1990er Jahre liegt die Hoffnung zugrunde, die Bevölkerung würde sich für Alltagsthemen wie Arbeitslosigkeit und soziale Gerechtigkeit mehr interessieren als für das Infragestellen des Holocausts.

Insbesondere in strukturell benachteiligten Regionen tritt die NPD als Speerspitze einer "Volksfront von rechts « und ihre Vorfeldorganisationen seitdem zunehmend als » Kümmerer « auf. Ihre Vertreter nehmen sich scheinbar der sozialen Sorgen der Menschen an, indem sie Freizeitangebote für Jugendliche, Hausaufgabenbetreuung, Beratungen zum Thema ALG II und ähnliche klassische Leistungen der Wohlfahrtsarbeit anbieten.

Besonders perfide wird der »Kampf um die Köpfe«, wenn die Bedürfnisse von Kindern und Jugendlichen nach Sicherheit, Verlässlichkeit, Wertschätzung, Zugehörigkeit und auch nach Abenteuer und Welterkundung benutzt werden, um Intoleranz, Ausländerfeindlichkeit und Rassismus zu verbreiten. Beispiel: Die »Heimattreue Deutsche Jugend (HDJ) Bund zum Schutz für Umwelt, Mitwelt und Heimat e. V.«. Die Organisation wurde 1990 als bundesweit aktiver Jugendverband gegründet und führte in den vergangenen Jahren Zeltlager, Kinderfeste und andere vorgeblich unpolitische Freizeitangebote durch. Im Oktober 2008 wurden bundesweit Durchsuchungen an nahezu einhundert Standorten durchge- 
Der Anstoß zu einem Fachkongress der Bundesarbeitsgemeinschaft der Freien Wohlfahrtspflege am 23. und 24. Oktober 2009 in Berlin über pädagogische und soziale Bestrebungen rechtsextremer Organisationen kam von Dr. Thomas de Maizière, Chef des Bundeskanzleramts und Bundesminister für besondere Aufgaben. In seinem Vortrag auf der Veranstaltung erläuterte er, was ihn zu dieser Anregung veranlasste und welche kritischen Punkte er beim gegenwärtigen Umgang mit rechtsextremistischen Einstellungen sieht. Auszüge aus seinem Grußwort:

Ich will zunächst ein Wort zur Notwendigkeit der Repression sagen: Es gibt keine Auseinandersetzung mit dem Rechtsextremismus, ohne dass nicht auch auf Repression, das heißt die konsequente Verfolgung und Ahndung jeder Form politisch rechtsextrem motivierter Kriminalität mit den Mitteln des Strafrechts, zurückgegriffen werden müsste. Das ist nötig und Teil unserer wehrhaften Demokratie. Gerade jugendlichen Tätern müssen - auch von den Eltern - frühzeitig klare Grenzen gesetzt werden. Die Täter müssen die Konsequenz und Entschlossenheit des Staates unmittelbar spüren. Unser Strafrecht bietet dafür das notwendige Instrumentarium. Richter und Staatsanwälte müssen es zwar mit Augenmaß, aber doch mit aller Konsequenz und vor allem schnell anwenden. Sie tun das auch in der Regel.

Das reicht aber nicht. Die Sicherheitsbehörden ergreifen zusätzlich eine Vielzahl von Maßnahmen, um Rechtsextremismus und politisch rechtsextrem motivierte Kriminalität in allen Erscheinungsformen zu bekämpfen. Neben der Tätigkeit der Verfassungsschutzbehörden nenne ich beispielhaft Maßnahmen wie Gefährderansprachen, verstärkte polizeiliche Präsenz an einschlägigen Treffpunkten, Auflösung von Konzerten, Demonstrationsverbote oder die Indizierung von entsprechenden Tonträgern. Auch Vereinsverbote halte ich, wenn sie sachgerecht erscheinen und so vernünftig vorbereitet sind, dass sie vor Gericht Bestand haben, für eine wichtige Maßnahme zur Bekämpfung des Rechtsextremismus. [...]

Der Bereich des `Kümmerns` muss stärker in den Blick genommen werden. Es sind Alternativen zu bieten, wenn zum Beispiel die NPD vor einem Autohaus in einem ländlichen Gebiet Ostdeutschlands eine Hüpfburg aufstellt. Wenn die NPD ein Freizeitangebot dieser Art unterbreitet - ich will in diesem Zusammenhang nicht von sniederschwellig sprechen, weil dieser Begriff anders verwendet wird; eher handelt es sich um ein schlichtes Freizeitangebot -, dann reicht es nicht aus, darüber empört zu sein. Man kann sicherlich das Autohaus und die NPD beschimpfen; das ist auch richtig so. Aber den Kindern zu sagen: 'Ihr dürft nicht in die Hüpfburg, weil die NPD sie aufgestellt hat‘, reicht nicht aus. Man stellt am besten eine andere Hüpfburg daneben. Das ist zwar eigentlich ein schlechtes Beispiel, weil man bessere, spannendere Freizeitaktivitäten anbieten sollte als eine Hüpfburg.

Was aber Freizeitangebote im ländlichen Bereich angeht - das ist meine Erfahrung -, beschimpfen wir die NPD oder ihre Tarnorganisationen dafür, dass sie sich kümmern, während wir nichts oder zu wenig dagegensetzen. Das ist ein Problem und führt auch eher zu einer Solidarisierung mit der NPD. Die Hüpfburg allein erscheint in den Augen vieler noch nicht als etwas, womit Kinder zu irgendetwas verleitet werden können. Es gibt das berühmte Lied 'Spiel nicht mit den Schmuddelkin- dern . Wenn diese aber als solche tabuisiert sind, sind sie besonders interessant. Mit der Tabuisierung >Das sind Schmuddelkinder, mit denen gehen wir nicht um^springt man zu kurz.

Viele von Ihnen werden jetzt einwenden, das sei auch eine Frage der sozialen Infrastruktur, insbesondere ihrer Kosten. Da müsse mehr geschehen. Viele der Argumente, die Sie sicherlich vortragen werden, sind richtig. Aber auch das reicht als Erklärung der Umstände nicht aus.

Ich kenne viele hervorragend hergerichtete, durchaus gut geführte und von den Öffnungszeiten her attraktive Jugendklubs, die aber leer sind. Ein Teil der problematischen Jugendlichen hängt doch an der Bushaltestelle herum. Es liegt oft nicht am Fehlen der Infrastruktur, das heißt des Jugendklubs an sich einschließlich Träger. Vielmehr wird eine bestimmte Form von Angeboten von einem bestimmten Teil der Jugendlichen nicht angenommen. Anders formuliert: Das Angebot, das diese Jugendlichen nutzen möchten, gibt es nicht im Jugendklub.

Noch einmal: Ich behaupte nicht, dass im Bereich der sozialen Infrastruktur nicht noch mehr getan werden müsste. Aber wenn wir schon auf einer Fachtagung miteinander sprechen und es nicht beim Austausch großer Deklarationen bewenden lassen wollen - so bitte ich mein Grußwort auch zu verstehen -, dann müssen wir über die Forderung nach mehr Geld hinauskommen und die Frage stellen: Wie ist die Qualität unseres Angebots? Erreichen wir die richtigen Leute? Die ‘Kümmerkompetenz all derer aus dem rechtsextremistischen $\mathrm{Mi}$ lieu, die sich vor Ort tummeln, wird nicht in erster Linie durch Beschimpfung der Kümmerer - obwohl auch das notwendig ist -, sondern vor allem durch kluge, gute Alternativangebote, und zwar in der ganzen notwendigen Breite, wirksam bekämpft. [...]

Ich möchte Ihnen einen weiteren Eindruck schildern: In unserer Gesellschaft bemühen wir uns oft zu sehr um diejenigen, die unsere Hilfe eigentlich nicht brauchen. Wenn Aktionen gegen >Pro Köln< initiiert werden oder in Dresden gegen den Versuch der NPD vorgegangen wird, das Gedenken an die Bombardierung der Stadt zu missbrauchen, dann ist das Ausdruck eines wunderbaren, bewegenden bürgerschaftlichen Engagements und von Zivilcourage.

Das ist aber, wenn man so will, nur eine Frage der Mobilisierung. Die Gefährdeten - um diese geht es eigentlich - werden aber nicht erreicht. Wer arbeitet mit denen, die schon halb unterwegs sind, die von den Angeboten der Rechtsextremisten schon halb gefangen sind? [...] Ist das eine Stellenfrage oder eine Qualitätsfrage? Haben Sie die Leute, die bereit sind, in diese Szene zu gehen? Ist das wirklich ein Geldproblem oder will man sich mit der Szene selbst lieber nicht auseinandersetzen? Kommt es im beruflichen Umfeld gut an bzw. wird es als >cook angesehen, wenn ein Sozialarbeiter auf die Frage > Was machen Sie denn? < antwortet: >Ich arbeite mit rechtsextremen Jugendlichen<? Im Fußball gibt es aber den Ausspruch: Man muss dahin gehen, wo es wehtut.

Der vollständige Wortlaut des Grußwortes von Dr. Thomas de Maizière kann in der Online-Dokumentation der Fachtagung eingesehen und kostenlos heruntergeladen werden (Internet http://www.bagfw.de/?id=104001000098). 
führt. Im Mai 2009 hat der Bundesinnenminister die »HDJ « verboten.

Die konsequente Reaktion von Exekutive und Judikative ist notwendig und wünschenswert, aber nicht hinreichend. Die Erfahrungen - keineswegs nur in den östlichen Bundesländern - zeigen: Die Freie Wohlfahrtspflege muss gemeinsam
Rechtsextremen in den Wohlfahrtsverbänden verhindert und Unterwanderungen von Einrichtungen und Diensten erkannt und vermieden werden können. Ebenso wurde beraten, welche Hilfen für jungen Menschen notwendig sind, um aus der rechtsextremen Szene aussteigen zu können*.

\section{»Die Freie Wohlfahrtspflege muss noch aufmerksamer werden "}

mit anderen zivilgesellschaftlichen Organisationen noch aufmerksamer werden. Die vermeintliche soziale »Kümmerpolitik « rechtsextremer Organisationen dient nicht der Stärkung der Interessen aller sozial benachteiligten Menschen ohne Ansehen ihrer Herkunft. Sie ist Mittel zum Zweck der »Nationalisierung des Sozialen .

Vor diesem Hintergrund einer Intensivierung des sozialen Engagements durch rechtsextremistische Organisationen beschlossen die Spitzenverbände der Freien Wohlfahrt im Februar 2007 eine intensive Auseinandersetzung mit dem Thema Rechtsextremismus. Den Auftakt dazu bildete eine im Oktober 2008 durchgeführte Veranstaltung der Bundesarbeitsgemeinschaft der Freien Wohlfahrtspflege in Berlin mit dem Titel »Freie Wohlfahrt gegen pädagogische und soziale Bestrebungen rechtsextremer Organisationen «. Ziel des Kongresses war es, die Aktivitäten und Strategien rechtsextremer Organisationen $\mathrm{zu}$ thematisieren und in den Fokus der Sozialen Arbeit zu rücken. Kommunalpolitische Gegenstrategien sollten entwickelt werden und aktuelle Ansätze der Gemeinwesenarbeit und der Jugendarbeit zur Sprache kommen. Erörtert wurde, wie Mitgliedschaften von

\footnotetext{
* Die Dokumentation der Tagung »Freie Wohlfahrt gegen pädagogische und soziale Bestrebungen rechtsextremer Organisationen « und ein Überblick über die weiteren Aktivitäten der Bundesarbeitsgemeinschaft der Freien Wohlfahrtspflege gegen Rechtsextremismus können auf der Webseite der Organisation eingesehen werden (Internet http://www.bagfw.de).
}

In seiner Eröffnungsrede zu dieser Veranstaltung kündigte der damalige Präsident der Bundesarbeitsgemeinschaft der Freien Wohlfahrtspflege, Prälat Dr. Peter Neher, weitere Maßnahmen der Freien Wohlfahrt in diesem Kontext an. Ein erster Schritt dazu wurde mit der Gründung einer gemeinsamen Arbeitsgruppe "Rechtsextremismus « innerhalb der Bundesarbeitsgemeinschaft der Freien Wohlfahrtspflege unternommen, die weitere verbandsübergreifende Aktivitäten und Maßnahmen koordinieren wird.

Aus der Überzeugung heraus, eine erfolgreiche Auseinandersetzung mit dem Rechtsextremismus bedürfe einer kommunalen Verankerung, sieht das Konzept der Arbeitsgruppe in zentralen Punkten eine Zusammenarbeit der Wohlfahrtsverbände mit den Kommunen vor. Kernstück der geplanten Maßnahmen ist daher eine Empfehlung gegen soziale Aktivitäten rechtsextreme Organisationen, welche die Bundesarbeitsgemeinschaft der Freien Wohlfahrtspflege gemeinsam mit dem Deutschen Verein für öffentliche und private Fürsorge erarbeiten wird. Verknüpft mit gezielten Fortbildungsangeboten, soll die Empfehlung die Fachkräfte vor Ort im Hinblick auf die notwendige Auseinandersetzung mit dem Rechtsextremismus sensibilisieren und mit dem geeigneten Rüstzeug versehen.

Das Thema Rechtsextremismus stellte in der Vergangenheit kein zentrales Thema für die Freie Wohlfahrtspflege dar. Nun haben die Spitzenverbände der Freien Wohlfahrtspflege die Zeichen der Zeit erkannt und sie werden sich auf Bundes-, Landes- und kommunaler Ebene verstärkt mit diesem Thema auseinandersetzen.

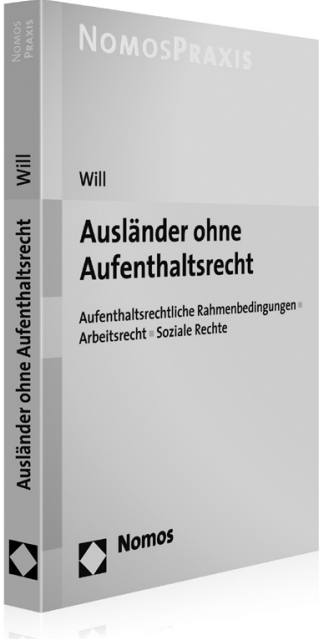

\section{Ausländer ohne Aufenthaltsrecht}

Aufenthaltsrechtliche Rahmenbedingungen | Arbeitsrecht | Soziale Rechte Von RAin Prof. Dr. Annegret Will 2008, 296 S., brosch., 39,- $€$, ISBN 978-3-8329-2500-0

Für die Beratung von Ausländern, die kein Aufenthaltsrecht (mehr) oder nur ein prekäres Aufenthaltsrecht besitzen, sind Kenntnisse des Aufenthalts-, Arbeits- und Sozialrechts ebenso unentbehrlich wie straf- und datenschutzrechtliches Wissen.

Das Handbuch behandelt sämtliche Rechtsfragen, die sich in der Beratungsarbeit stellen.

"ein wertvolles Hilfsmittel, das zu einem wirklichen Vademecum in der Beratung von Illegalisierten werden sollte."

Prof. Dr. Winfried Möller, NDV-Nachrichten 7/08

\section{Nomos}

Bitte bestellen Sie im Buchhandel oder versandkostenfrei unter $\downarrow$ www.nomos-shop.de 